\title{
Chronic intestinal pseudoobstruction and ophthalmoplegia in a patient with mitochondrial myopathy
}

\author{
R CERVERA, J BRUIX, A BAYES, R BLESA, I ILLA, J COLL, \\ AND A M GARCIA-PUGES \\ From the Gastroenterology, Hepatology and Neurology Services, Hospital Clínic i Provincial, Neurology \\ Service, Hospital de Sant Pau, Barcelona, Spain.
}

\begin{abstract}
SUMMARY A 38 year old woman having chronic intestinal pseudoobstruction associated with mitochondrial myopathy is reported. The clinical and radiographic features suggested the diagnosis of chronic intestinal pseudoobstruction. Muscular atrophy and ophthalmoplegia led to muscle biopsy, which disclosed accumulation of normal and abnormal mitochondria ('ragged red fibres'), characteristic of mitochondrial myopathy.
\end{abstract}

Intestinal pseudoobstruction is a clinical syndrome caused by ineffective intestinal propulsion. It is characterised by symptoms and signs of mechanical bowel obstruction in the absence of an occluding lesion of the intestinal lumen. The most common type is the acute variety, which is usually self-limited and related to surgical procedures or acute illnesses. Chronic intestinal pseudoobstruction is much less common and it may be idiopathic or secondary to a known systemic disease.$^{1-3}$ It has been also described associated with several myopathies, such as myotonic dystrophy ${ }^{4}$ familial visceral myopathy ${ }^{5}$ and dermatomyositis. ${ }^{6}$

The association of chronic intestinal pseudoobstruction and ophthalmoplegia has recently been documented in patients having a familial visceral myopathy. ${ }^{7}$ Ophthalmoplegia is a striking but nonspecific clinical sign which occurs in different diseases. In some instances, it is part of a well-defined neuromusclar disorder, for example, myasthenia gravis, thyrotoxicosis, myotonic dystrophy, and mitochondrial myopathy. ${ }^{\circ}$

We report here the clinical, laboratory, radiographic, manometric, and pathologic findings of a

Address for correspondence: Dr J Bruix, Liver Unit, Hospital Clinic i Provincial, Cl. Villarroel 170, 08036-Barcelona, Spain.

Received for publication 9 October 1987. patient having a chronic intestinal pseudoobstruction and ophthalmoplegia, whose muscle biopsy disclosed a mitochondrial myopathy.

\section{Case report}

A 36 year old white woman was admitted to hospital in February 1985 for evaluation of intermitent attacks of abdominal pain associated to distension, nausea, vomiting, and non-specific diarrhoea of two to seven days duration, and variable intensity, which started when she was 28 years old. She was the first of two siblings. Her parents and sister were alive and in good health. She was a product of an apparently normal full term pregnancy. No abnormality was noted in the perinatal period and her psychomotor development was considered normal. She developed constipation during childhood and at age 23 she noted impairing of the visual acuity and a diagnosis of myopia was made. At age 28 she complained of progressive hearing loss and four years before hospitalisation she became amenorrhoeaic.

On admission she was oriented, alert, and of normal average intelligence. She was $154 \mathrm{~cm}$ tall and weighed $33 \mathrm{~kg}$. Her blood pressure was $110 / 85 \mathrm{mmHg}$ and her pulse rate was 68 beats per minute. Lungs were clear and the heart was not enlarged, with 544 


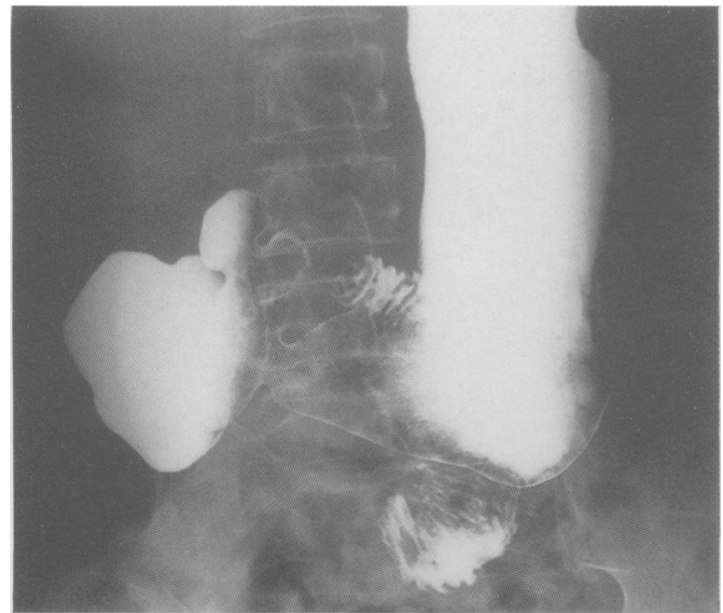

Fig. 1 Uppergastrointestinal series showing evidence of gastroparesia, megaduodenum, and slight dilatation of the small intestine.

regular rhythm. No murmur or pericardial friction rub was heard. Abdomen was distended and no visceromegaly was found.

Routine laboratory tests including serum creatinine, creatinine phosphokinase and aldolase were normal. Latex fixation test for rheumatoid factor, antinuclear antibody and anti-DNA antibody were negative. Complement factors were normal.

An electrocardiogram and chest radiographs were normal. An upper gastrointestinal series exhibited absence of oesophageal peristalsis, gastroparesis with delayed emptying, megaduodenum and diffuse dilatation of the rest of the small intestine (Fig. 1). The barium enema was normal. Oesophageal manometry revealed oesophageal aperistalsis and marked spontaneous and repetitive contractions throughout the oesophagus. The lower oesophageal sphincter pressure was normal. There were no abnormalities in intestinal absorption, the D-xylose test was normal. Serum T3, T4, and TSH concentrations were within normal range.

The neurologic examination showed normal pupils that responded well to light and accomodation. Ocular movements were restricted to a few degrees in any direction. Slight ptosis of the lids was noted. Ophthalmoscopic examination did not reveal retinal pigment, optic atrophy or arterial narrowing. Hearing was diminished bilaterally, with air conduction better than bone conduction. Deep tendon reflexes were normal, except for knee reflex that was diminished. There was generalised muscular atrophy and no fasciculations were observed. Cerebrospinal fluid glucose concentration was $63 \mathrm{ml} / \mathrm{dl}$, protein

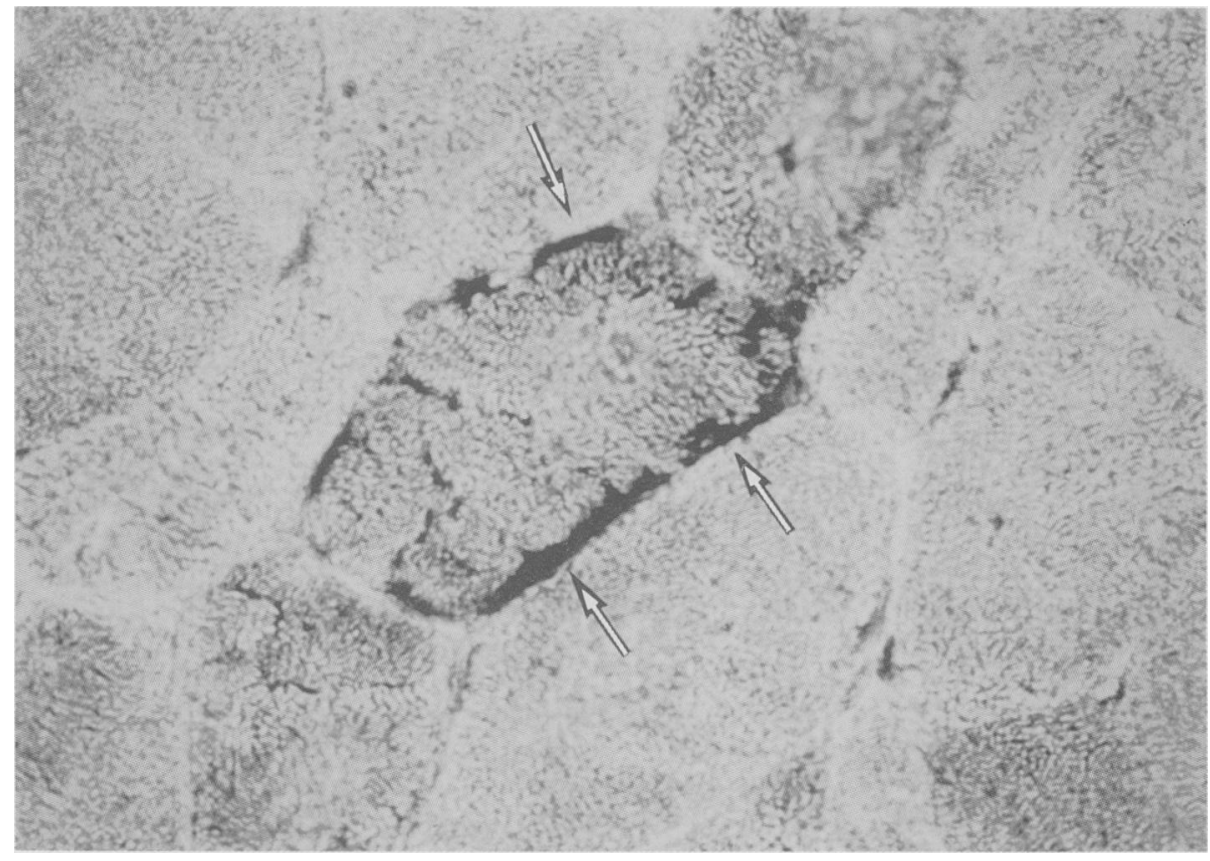

Fig. 2 Muscle biopsy stained with NADH oxidase technique. Subsarcolemmal aggregates of normal and abnormal mitochondria $(\rightarrow)$ are apparent. 


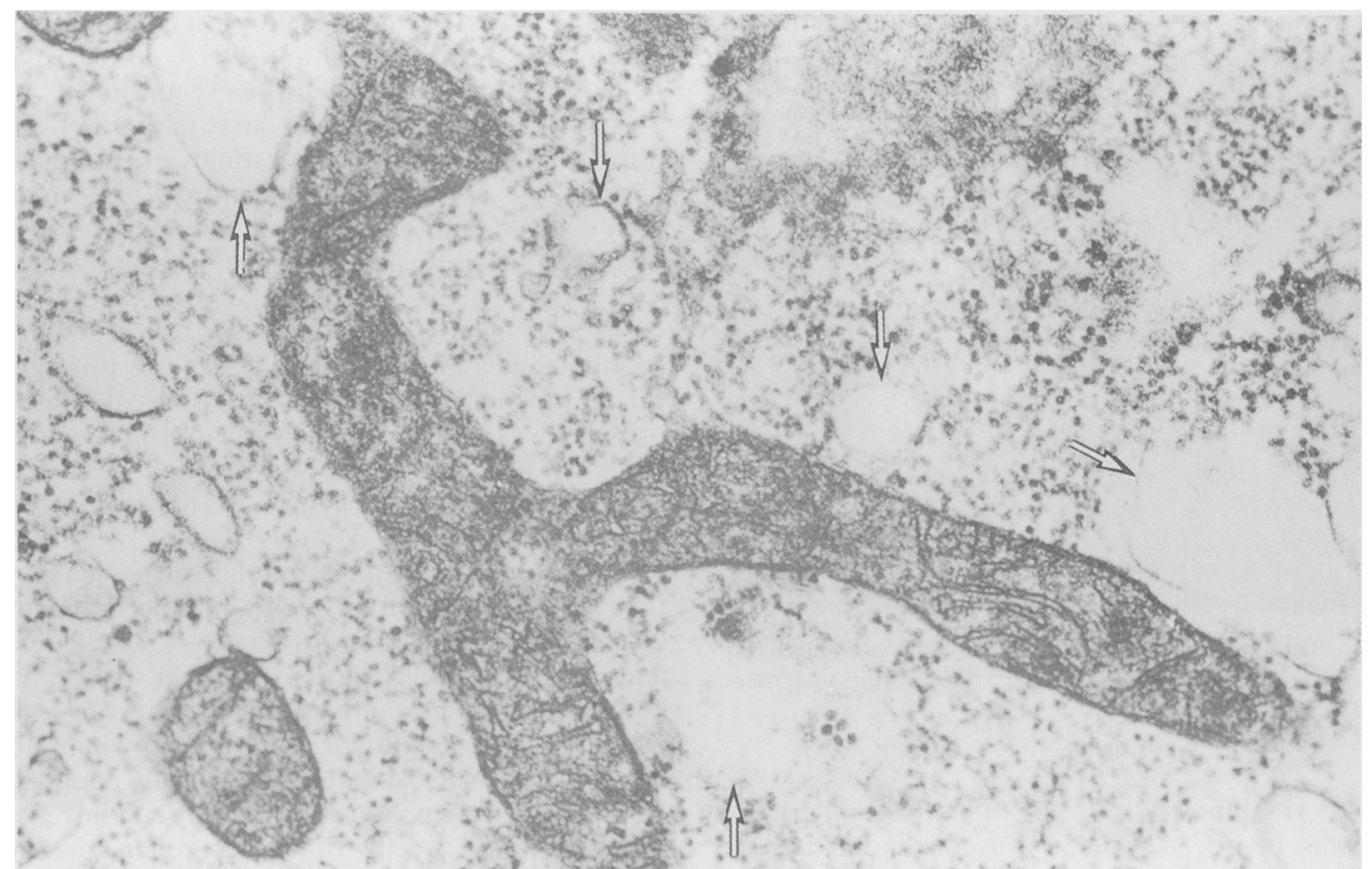

Fig. 3 Electron microscopy evidences abnormal mitochondria and excessive lipid droplets $(\rightarrow)$.

concentration was $47 \mathrm{mg} / \mathrm{dl}$ and no cells were found. The electromyogram showed polyphasic, short and small units, and fibrillations, being consistent with myopathy. The left deltoid muscle biopsy disclosed accumulation of excessive lipid droplets and normal and abnormal mitochondrias previously described as 'ragged red fibres' (Figs 2 and 3), setting the diagnosis of mitochondrial myopathy."

\section{Discussion}

Mitochondrial myopathies are a group of disorders of unknown aetiology in which the earliest or most striking morphological and histochemical changes occur in mitochondria. In none of these disorders, however, has it been unequivocally documented that the primary abnormality is located within the mitochondria. ${ }^{411}$

Several pathologic and clinical variations have been described among patients with mitochondrial myopathies. The patient here described fits the characteristics of those reported by Olsen et al in 1972. These authors grouped seven cases in which the histochemical and ultrastructural examination showed excessive lipid droplets and accumulation of normal and abnormal mitochondria, in the striated muscles. This was the sole or major abnormality and was named 'ragged red fibres'. All these seven patients had progressive ophthalmoplegia and some of them had additional features such as raised cerebrospinal fluid protein levels, cardiac conduction abnormalities, retinal pigmentation, impaired growth, sensorineural hearing defect, abnormal electroencephalogram, and ataxia. This disorder was refered by the authors as 'oculocraniosomatic neuromuscular disease with ragged-red fibres'. ${ }^{8}$ Other mitochondrial myopathies reported as Kearns-Sayre syndrome, myoclonus epilepsy with ragged-red fibres, and MELAS syndrome (mitochondrial myopathy, encephalopathy, lactic acidosis, strokelike episodes) probably are variants of this disease." Neurological abnormalities such as ophthalmoplegia and hearing loss, as seen in our patient, are part of this syndrome but no definitive explanation has been found. Postmortem examination in a few patients, however, has disclosed a diffuse coarse vacuolation of cerebral tissue, particularly of the parts concerned with eye movements."

The main clinical manifestation of our patient was chronic intestinal pseudoobstruction. This abnormality has been described associated with collagen disease, endocrine disorders, and other systemic diseases. ${ }^{2}$ It has also been related with several myopathies, such as myotonic dystrophy, ${ }^{4}$ familial visceral myopathy, and dermatomyositis. ${ }^{\circ}$ Anuras et al, ${ }^{7}$ have recently reported the presence of chronic 
intestinal pseudoobstruction in association with ophthalmoplegia in two patients having a familial visceral myopathy. This disorder has an autosomal recessive transmission and the intestinal smooth muscle appears to be the primary area damaged, resulting in a characteristic vacuolar appearance. This clinical and pathologic pattern makes it a different syndrome from the one reported by us, despite the lack of intestinal biopsy in our patient. It was not done because the diagnosis of chronic intestinal pseudoobstruction was firmly established by the clinical, laboratory, radiographic, and manometric findings, and it is known that intestinal biopsy is not without risk in patients with this disease.

In summary, chronic intestinal pseudoobstruction enlarges the spectrum of signs and symptoms associated with mitochondrial myopathies. This clinical association may suggest a possible role of mitochondrial myopathies in the development of chronic intestinal pseudoobstruction, and therefore, this rare disease could be included in the aetiological diagnosis of patients with chronic intestinal pseudoobstruction.

\section{References}

1 Schuffler MD, Deitch EA. Chronic idiopathic intestinal pseudoobstruction. A surgical approach. Ann Surg 1980; 192: 752-61.
2 Schuffler MD, Rohrmann CA, Chaffee RG, Brand DL, Delaney JH, Young JH. Chronic intestinal pseudoobstruction. A report of 27 cases and review of the literature. Medicine (Balt) 1981; 60: 173-96.

3 Snape WJ Jr. Pseudoobstruction and other obstructive disorders. Clin Gastroenterol 1982; 11: 593-608.

4 Goldberg HI, Sheft DJ. Esophageal and colon changes in myotonia dystrophica. Gastroenterology 1972; 63: 134.

5 Faulk DL, Anuras S, Gardner GD, et al. A familial visceral myopathy. Ann Intern Med 1978; 89: 600-6.

6 Kletchner FS. Dermatomyositis and its manifestations in the gastrointestinal tract. Am J Gastroenterol 1970; 53: 141.

7 Anuras S, Mitros FA, Nowak TV, et al. A familial visceral myopathy with external ophthalmoplegia and autosomal recessive transmission. Gastroenterology 1983; 84: 346-53.

8 Olson W, Engel WK, Walsh GO, Einaugler R. Oculocraniosomatic neuromuscular disease with "ragged-red" fibers. Arch Neurol 1972; 26: 193-211.

9 Spiro AJ, Prineas JW, Moore CL. A new mitochondrial myopathy in a patient with salt craving. Arch Neurol 1970; 22: 259-69.

10 Adams RD, Victor $M$. The congenital neuromuscular disorders. In: Principles of neurology New York: McGraw-Hill, 1985.

11 DiMauro S, Bonilla E, Zeviani M, Nakagawa M, De Vivo DC. Mitochondrial myopathies. Ann Neurol 1985; 15: 521-38. 